\title{
Spectrally Efficient SSB signals for W-band Links Enabled by Kramers-Kronig Receiver
}

\author{
Luis Gonzalez Guerrero ${ }^{1 *}$, Haymen Shams ${ }^{1}$, Irshaad Fatadin², Martyn J. Fice ${ }^{1}$, Mira Naftaly ${ }^{2}$, Alwyn J. \\ Seeds ${ }^{1}$ and Cyril C. Renaud ${ }^{1}$ \\ ${ }^{1}$ Department of Electronic \& Electrical Engineering, University College London, London, WC1E 7JE, UK \\ ${ }^{2}$ Electromagnetic Technologies group, National Physical Laboratory, London, TW11 OLW, UK \\ *uceelgo@ucl.ac.uk
}

\begin{abstract}
We demonstrate a radio-over-fiber link based on the Kramers-Kronig receiver for the first time. Using this technique, we recover an $11 \mathrm{GBd}$ single sideband signal with a net spectral efficiency of $3.4(\mathrm{bit} / \mathrm{s}) / \mathrm{Hz}$ at W-band.

OCIS codes: (060.2330) Fiber optics communications; (060.5625) Radio frequency photonics; (130.0250) Optoelectronics
\end{abstract}

\section{Introduction}

Radio-over-fiber (RoF) techniques based on heterodyning two lasers in a photodiode offer great prospects, in terms of cost and power consumption, for the development of future backhaul wireless links [1]. A drawback of photonic mixing is that the resulting wireless signal exhibits a phase noise equal to the sum of the two laser linewidths. While external cavity lasers (ECLs) can offer narrow linewidths, they are significantly bulky and quite expensive, and are best suited for laboratory use exclusively. For industrial deployment, low cost free running lasers (with linewidths > $1 \mathrm{MHz})$ are preferable. In this case, the phase noise can prevent the coherent receiver ( $\mathrm{Rx}$ ) from recovering the transmitted signal [2]. A potential solution to this is the use of phase noise-robust modulation formats, such as single sideband (SSB) modulation, together with envelope detection.

In a recent paper, we have demonstrated the phase noise insensitivity of this scheme in a RoF system using two broad-linewidth free-running lasers [3]. Apart from enabling the use of low cost lasers, this scheme also relaxes the complexity of the digital signal processing (DSP) operations aimed at recovering the phase of the transmitted signal. Furthermore, a receiver based on envelope detection, unlike those based on mixing, does not require a local oscillator, further simplifying the system architecture.

One of the issues with SSB envelope detected signals, is the guard band they require between carrier and sideband to combat the signal-signal beat interference (SSBI), which results from the overlap of the direct detection (DD) terms and the signal. This guard band reduces the spectral efficiency and may prevent the use of this scheme in situations where limited spectrum is available and high data rates are required. To enable the use of narrow guard bands, a number of DSP algorithms have been proposed that try to estimate and eliminate the DD products.

Recently, a scheme based on the Kramers-Kronig (KK) relations has been proposed for DD optical networks as a means to combat the SSBI [4]. As long as the SSB transmitted signal has a sufficiently high carrier-to-sideband power ratio (CSPR), this scheme can reconstruct the optical phase of the signal reaching the envelope detector, effectively eliminating the DD terms. It has been demonstrated that this technique has the best SSBI cancellation performance in optical systems [5]. In this paper we use, for the first time, the KK scheme in a RoF system working at $100 \mathrm{GHz}$. First, we compare its performance with the case where no KK algorithm is used. Then, we study the performance of both schemes with increasing guard band, and finally we use the KK receiver to transmit and detect an 11 GBd 16 QAM signal with no guard band.

\section{Experimental arrangement}

The complete experimental arrangement is shown in Fig. 1. In the receiver, we used a harmonic mixer instead of an envelope detector in order to measure the CSPR, which is a critical parameter in the SSB modulation format. The envelope detection is here implemented in the digital domain after analog-to-digital conversion. We expect, nevertheless, the results obtained here to be applicable to systems using an analog envelope detector.

For digital signal generation, four $2^{11}$ de Bruijn bit sequences were mapped into 16-QAM symbols at a symbol rate of $7.5 \mathrm{GBd}$. After bit-to-symbol mapping, a root raised cosine (RRC) filter with a roll-off factor of 0.1 was applied to the $I$ and $Q$ components. This baseband signal was then digitally up-converted to a frequency equal to $4.125 \mathrm{GHz}$ ( 0.55 times the symbol rate) to achieve a SSB signal with no guard band. Finally, to remove the lower sideband, a Hilbert transform (HT) was applied to the resultant waveform. To ensure identical time delay in both $I$ and $Q$ paths, two phase shifters were placed after the arbitrary waveform generator (AWG) and before the electrical amplifiers, as shown in Fig. 1. 
On the transmitter side, an ECL was used for data modulation. The optical signal was then combined with a second ECL after transmission through $10 \mathrm{~km}$ of optical fiber. The two optical tones, set at a frequency difference of $100 \mathrm{GHz}$, were sent to a uni-travelling carrier photodiode (UTC-PD) after optical amplification and filtering. Fig. 1 inset (a) shows the combined optical signals before the UTC-PD.

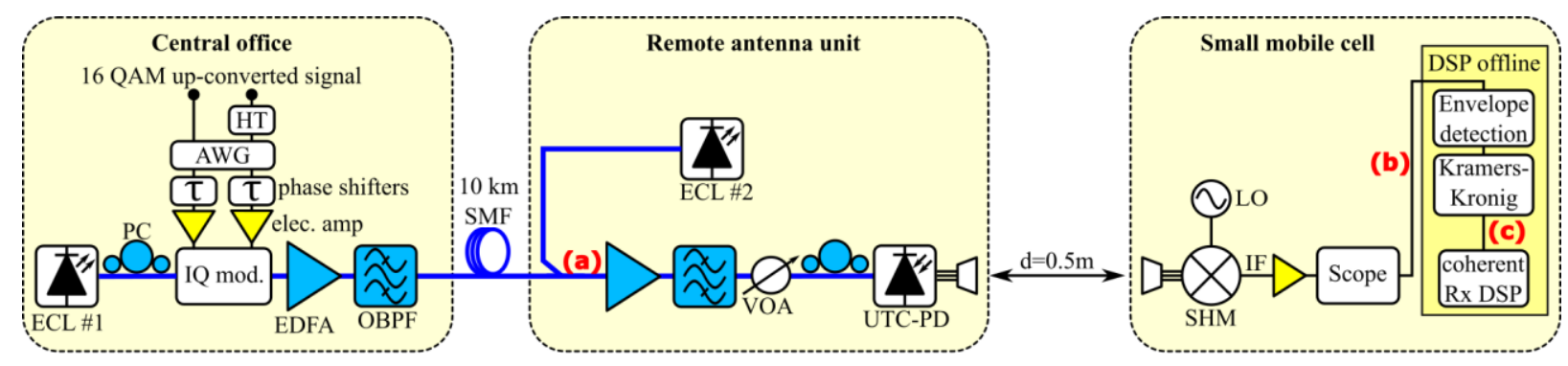

(a)

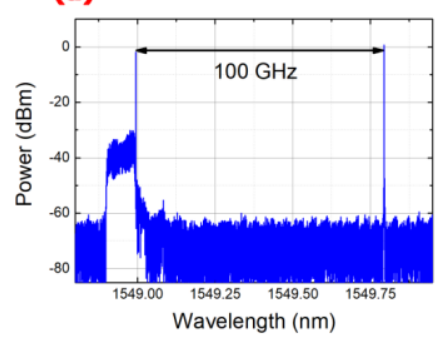

(b)

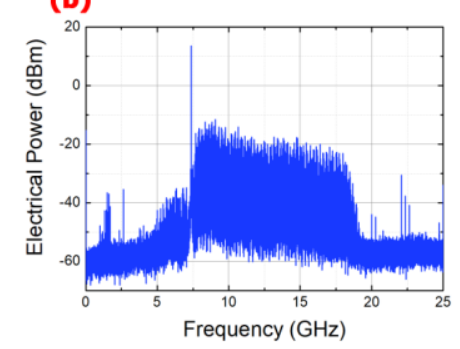

(c)

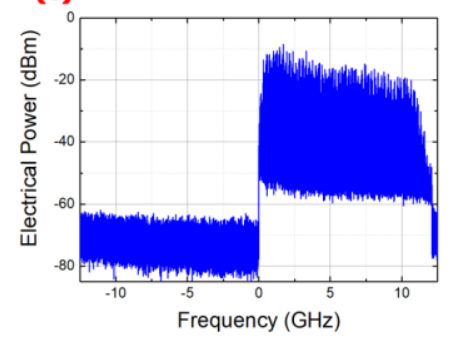

Fig. 1. Experimental system depicting the arrangement expected in wireless backhaul links based on RoF. Insets: (a) combined optical signals before the UTC-PD, (b) received SSB signal at intermediate frequency, and (c) signal after KK receiver.

After wireless propagation of $0.5 \mathrm{~m}$ the received signal was down-converted to an $\mathrm{IF}$ of around $11 \mathrm{GHz}$ by mixing it with the output of an RF synthesizer in a second-harmonic mixer (SHM). The down-converted signal was then sampled by an $80 \mathrm{GSa} / \mathrm{s}$ oscilloscope. Following analog-to-digital conversion, envelope detection and down sampling to $5 \mathrm{Sa} /$ symbol, the KK algorithm was performed by applying a HT to the sampled signal (for a detailed description of the algorithm see [5]). Finally, a typical coherent DSP routine consisting of IQ down-conversion, matched filtering, resampling, normalization, and equalization was carried out. The equalizer was initialized using the radius directed algorithm (RDE). Then the Viterbi-Viterbi algorithm and a block averaging filter of 600 samples in length was applied for phase corrections before switching to a decision-directed (DD) mode equalizer. Finally, symbol decision and bit error counting were performed after grey decoding.

\section{Results}

In direct-detection SSB systems using small guard bands, there is a trade-off between the SSBI and the additive white Gaussian noise (AWGN). Signals with low CSPR ratio suffer from high SSBI, while high CSPR leads to large AWGN penalties. Due to their ability to suppress the SSBI products, cancelation techniques allow operation at lower values of CSPR (ideally at $0 \mathrm{~dB}$, which is the optimum CSPR when the SSBI products do not overlap with the desired signal [6]). To assess the reduction of CSPR enabled by the KK receiver, the bit error rate (BER) was measured for different values of the CSPR (which was varied by adjusting the $I Q$ modulator biasing points) for the cases with and without the KK receiver. For this and the subsequent comparisons, $7.5 \mathrm{GBd}$ signals were used, giving a gross rate of $30 \mathrm{Gbit} / \mathrm{s}$. As can be seen in Fig. 2 (a), a reduction in the optimum CSPR of more than $7 \mathrm{~dB}$ was measured with the KK technique, confirming its ability to suppress the SSBI.

Next, to measure the improvement in receiver sensitivity, the BER was measured as a function of the square of the average photocurrent generated in the UTC-PD (this parameter being proportional to the generated RF power). As can be seen in Fig. 2 (b), only with the KK receiver, a BER below the hard-decision forward error correction (HD-FEC) threshold (BER of $3.8 \cdot 10^{-3}$ ) could be achieved.

We also characterized the performance of the system for different guard bands. In Fig. 2 (c) the BER is plotted against the normalized gap (the guard band divided by the signal bandwidth). With the KK receiver, a slight improvement was observed when the normalized gap was increased up to approximately 0.3. On the other hand, without the KK receiver, a clear improvement of the BER performance together with a reduction of the optimum CSPR were observed for increasing guard bands. However, even for the highest value of guard band tested in this 
paper (limited by transmitter bandwidth), the system without the KK receiver could not match the performance of the KK receiver.

Finally, we transmitted an 11 GBd 16 QAM signal (gross bit rate of $44 \mathrm{Gbit} / \mathrm{s}$ ) with no guard band and a roll-off factor of 0.1 , achieving a net spectral efficiency of $3.4(\mathrm{bit} / \mathrm{s}) / \mathrm{Hz}$ (taking into account the $7 \%$ overhead of the HDFEC). A BER of $3.1 \cdot 10^{-3}$, which is below the HD-FEC limit, was obtained. The received constellation diagram is shown in Fig. 3.

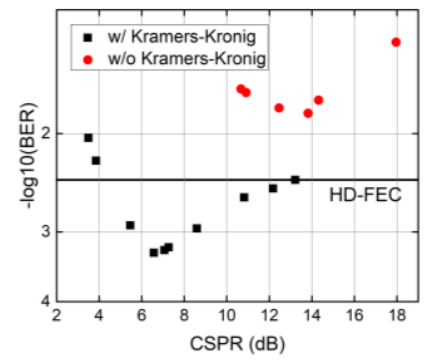

(a)

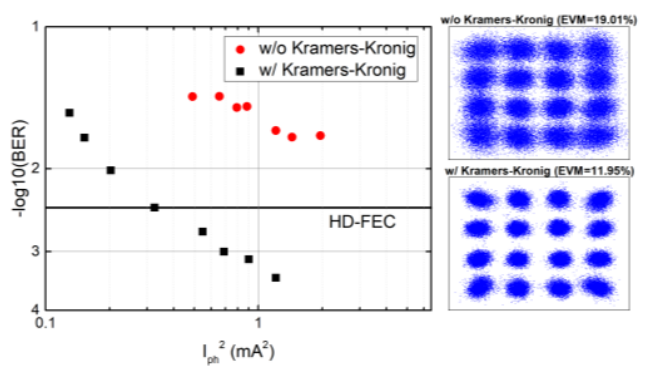

(b)

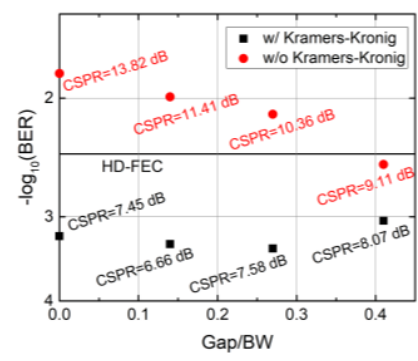

(c)

Fig. 2. (a) BER versus CSPR with and without the KK receiver; (b) BER vs. photocurrent squared with and without the KK receiver (the received constellation diagrams for the lowest value of BER in each case are shown at the right); (c) the BER as a function of the ratio between the guard band and the signal bandwidth (labels for each point indicate the optimum CSPR value).

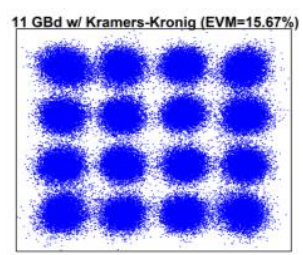

Fig. 3. The received constellation diagram for the 11 GBd 16 QAM signal.

\section{Conclusions}

We have demonstrated a SSB RoF link using a KK receiver for the first time. Compared with the conventional SSB receiver, the KK reduces the optimum CSPR by more than $7 \mathrm{~dB}$ and allows successful recovery of a 16 QAM 7.5 GBd SSB signal, which was not possible without this technique. Furthermore, we found that this scheme outperforms the system without KK for all normalized gaps tested in this paper (up to 0.4 approximately). Using the KK receiver, we have also transmitted an $11 \mathrm{GBd} 16$ QAM (44 Gbit/s) SSB signal with a net spectral efficiency of $3.4(\mathrm{bit} / \mathrm{s}) / \mathrm{Hz}$. All this is done using a simple system based on two free-running lasers and envelope detection.

\section{Acknowledgment}

The authors would like to acknowledge Frédéric van Dijk of Alcatel Thales III-V Lab for providing the UTC-PD. This work was supported by the EPSRC programme grant Coherent Terahertz Systems (COTS) (EP/J017671/1) and the project EMPIR 14IND13 PhotInd at NPL.

\section{References}

[1] A. J. Seeds, H. Shams, M. J. Fice, and C. C. Renaud, “TeraHertz photonics for wireless communications,” J. Light. Technol., vol. 33, no. 3, pp. 579-587, 2015.

[2] H. Shams, L. Gonzalez-Guerrero, M. Fice, Z. Yang, C. Renaud, and A. Seeds, "Distribution of multiband THz wireless signals over fiber," in Proc. SPIE 10128, Broadband Access Communication Technologies XI, 2017, vol. 101280G.

[3] L. G. Guerrero, H. Shams, J. Martyn, A. J. Seeds, C. C. Renaud, I. Fatadin, and M. Naftaly, "Experimental Investigation of Phase Noise

Tolerance of SSB THz Signals," in Topical Meeting on Microwave photonics (MWP), 2017, pp. 1-4.

[4] M. S. Antonio Mecozzi, Cristian Antonelli, "Kramers - Kronig coherent receiver," Optica, vol. 3, no. 11, pp. 1220--1227, 2016.

[5] Z. Li, M. S. Erkilinc, K. Shi, E. Sillekens, L. Galdino, B. C. Thomsen, P. Bayvel, and R. I. Killey, "SSBI mitigation and the kramers-kronig scheme in single-sideband direct-detection transmission with receiver-based electronic dispersion compensation," J. Light. Technol., vol. 35 , no. 10, pp. 1887-1893, 2017.

[6] W.-R. Peng, X. Wu, K.-M. Feng, V. R. Arbab, B. Shamee, J.-Y. Yang, L. C. Christen, A. E. Willner, and S. Chi, "Spectrally efficient directdetected OFDM transmission employing an iterative estimation and cancellation technique," Opt. Express, vol. 17, no. 11, pp. 9099-9111, 2009. 